\title{
Novel Physiological Functions of Branched-Chain Amino Acids
}

\author{
Yoshiharu SHImomura, Yasuyuki KITAURA, Yoshihiro Kadota, \\ Takuya IsHIKAWA, Yusuke Kondo, Minjun Xu, Miki OTA, \\ Yukako MorISHITA, Jussiaea V. BARIUAN and Hongmin ZHEN \\ Laboratory of Nutritional Biochemistry, Graduate School of Bioagricultural Sciences, \\ Nagoya University, Nagoya 464-8601, Japan
}

\begin{abstract}
Summary Branched-chain amino acids (BCAAs) are essential amino acids for humans and are major building blocks of proteins. Recent studies indicate that BCAAs act not only as components of proteins, but also as nutrasignals. In this review, we summarize the findings of recent studies investigating the physiological functions of BCAAs in the regulation of protein and glucose metabolism and brain function.
\end{abstract}

Key Words branched-chain amino acid, protein and glucose metabolism, brain function

Branched-chain amino acids (BCAAs: leucine, isoleucine, and valine) are nutritionally indispensable (essential) amino acids for humans. We acquire these amino acids mainly as food proteins, in which the BCAAs account for $\sim 20 \%$ of the amino acids. The ingested BCAAs are used for protein synthesis in the body. The daily requirement for BCAAs in adults is estimated to be $85 \mathrm{mg} / \mathrm{kg}$ body weight (BW) (39, 20, and $26 \mathrm{mg} /$ $\mathrm{kg}$ BW for Leu, Ile, and Val, respectively) using methods such as indicator amino acid oxidation and 24-hour balance studies (1). The primary physiological function of BCAAs is as the building blocks of proteins; however, recent studies have contributed to the mounting evidence for other physiological functions. In this review, we summarize the current findings on the physiological functions of BCAAs, including those from our own studies.

\section{Regulation of BCAA Catabolism}

The main BCAA catabolic pathway occurs within mitochondria. The first 2 steps of the pathway are common to the 3 BCAAs and are especially important for the regulation of BCAA catabolism (2) (Fig. 1). The first step of the pathway is the reversible transamination of BCAAs catalyzed by branched-chain aminotransferase (BCAT) to produce corresponding branched-chain $\alpha$-ketoacids (BCKAs). The second step involves the oxidative decarboxylation of BCKAs catalyzed by the BCKA dehydrogenase (BCKDH) complex to produce corresponding CoA esters. Since this reaction is irreversible, it is a rate-limiting step in BCAA catabolism. Furthermore, the BCKDH complex is subject to covalent modification; $\mathrm{BCKDH}$ kinase (BDK) is responsible for inactivation of the complex by phosphorylation and BCKDH phosphatase reactivates the complex by dephosphorylation (2). Therefore, the enzyme activity of the BCKDH complex can be quickly changed in response to alterations in physiological conditions. Aberrations of the enzymes involved in the first two steps of BCAA catabolism have

E-mail: shimo@agr.nagoya-u.ac.jp a great impact on the circulating concentrations of BCAAs; a defect in the mitochondrial BCAT (BCAT2) gene of mice resulted in a markedly high level of plasma BCAAs (3), and BDK-knockout (KO) mice, in which the BCKDH complex in all tissues was almost fully activated, showed significantly low concentrations of plasma BCAAs (4).

\section{Regulation of Protein Metabolism by Leucine}

The mammalian target of rapamycin complex 1 (mTORC1) is a serine-threonine protein kinase that controls translation initiation and is responsive to cellular levels of free amino acids (5). mTORC1 phosphorylates and activates the protein kinase p70S6K1, which subsequently phosphorylates eukaryotic initiation factor 4B (eIF4B) and programmed cell death 4 (PDCD4), and phosphorylates eIF4E-binding protein 1 (eIF4E-BP1), thereby permitting eIF4E to associate with eIF4G to form eIF4F. Thus, mTORC1 stimulates the joining of mRNA to the $43 \mathrm{~S}$ preinitiation complex and has a global effect on protein synthesis. Furthermore, mTORC1 regulates cellular proteolysis by inhibiting the formation of autophagosomes (6). It is also known that leucine strongly activates mTORC1 (5). Taken together, these findings suggest that BCAAs (especially leucine) are strong stimulants for protein anabolism.

In this context, BCAAs are therapeutically used in patients with liver cirrhosis, in whom plasma concentrations of BCAAs and albumin are significantly decreased (7). It has been demonstrated that BCAA supplementation ameliorates plasma albumin levels in patients (7). Moreover, it is noteworthy that this treatment dramatically decreased the frequency of muscle cramp in patients (8), suggesting that BCAA supplementation may also improve muscle protein metabolism.

\section{Glucose Metabolism and BCAAs}

It has been demonstrated through both in vitro and in vivo studies that leucine and isoleucine promote glucose metabolism by stimulating glucose uptake into muscle tissues $(9,10)$. Furthermore, it was reported 


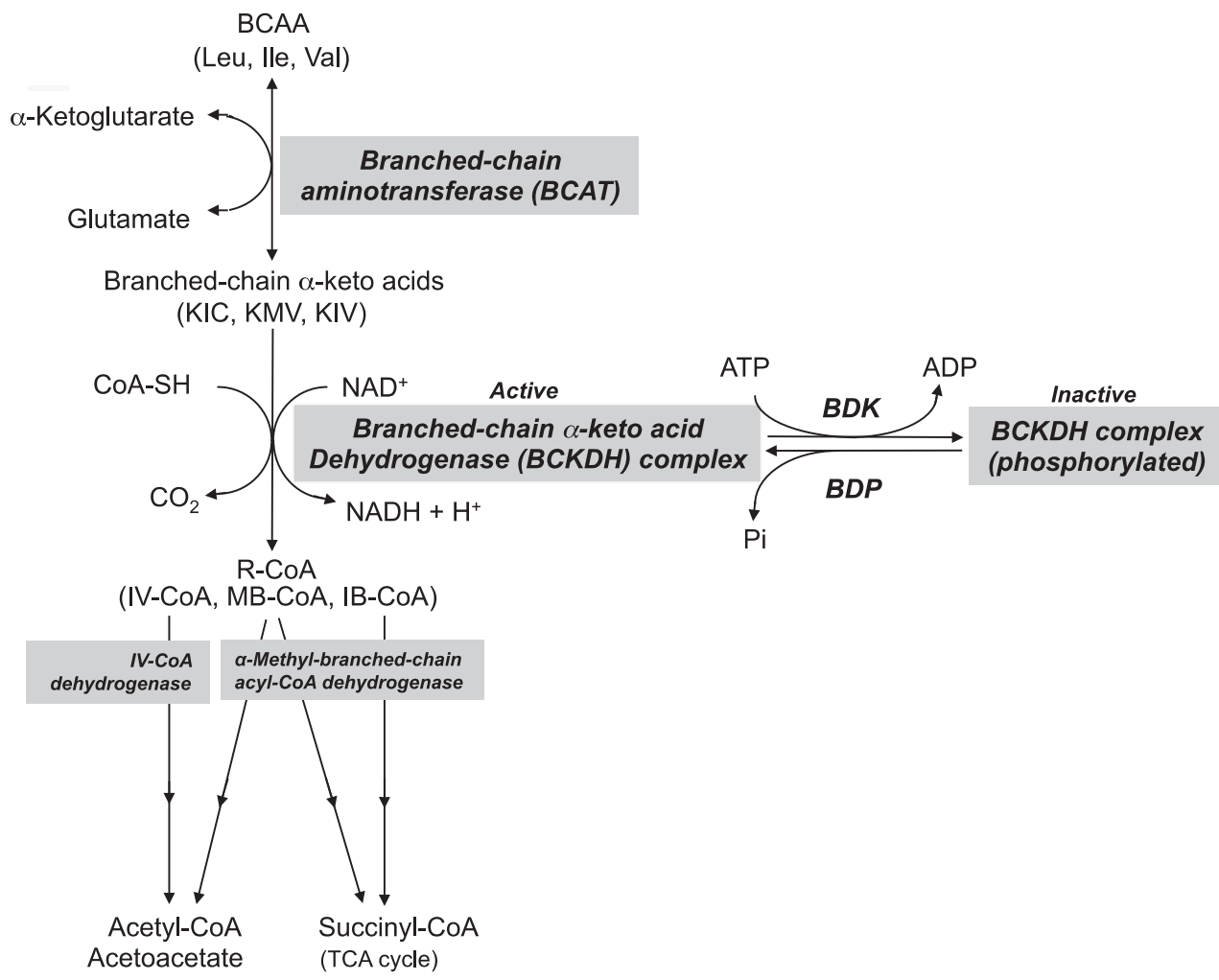

Fig. 1. BCAA catabolic pathway. BDK, BCKDH kinase; BDP, BCKDH phosphatase; KIV, $\alpha$-ketoisovalerate; KMV, $\alpha$-keto- $\beta$-methylvalerate; KIC, $\alpha$-ketoisocaproate; CoA-SH, coenzyme A, reduced form; IB-CoA, isobutyrylCoA; MB-CoA, $\alpha$-methylbutyryl-CoA; IV-CoA, isovaleryl-CoA; and R-CoA, acyl-CoA.

that BCAA supplementation improved glucose tolerance in a $\mathrm{CCl}_{4}$-induced liver cirrhosis rat model (11). A study of BCAT2-KO mice also showed that markedly increased plasma BCAAs significantly improved glucose tolerance and insulin sensitivity (3).

In order to examine whether alterations in the basal level of plasma BCAAs might affect glucose metabolism, we used clofibric acid (a hypolipidemic agent, administered as ethyl p-chlorophenoxyisobutyrate [clofibrate]), a BDK inhibitor, to activate the BCKDH complex, thereby resulting in decreased plasma BCAA concentrations in rats (12). The injection of clofibrate at $0.2 \mathrm{~g} / \mathrm{kg}$ BW decreased plasma BCAA concentrations to $~ 50 \%$ of the basal level for at least several hours. While the rats were in this condition, we conducted a glucose tolerance test. Rats with lowered plasma BCAA levels showed significant deterioration of glucose tolerance. However, the glucose tolerance deterioration in clofibrate-treated rats was recovered to the basal level by prior administration of BCAAs, which elevated plasma BCAA levels to basal levels. Furthermore, BDK-KO mice with lowered plasma BCAA concentrations ( $\sim 50 \%$ of basal $)$ showed significant deterioration of glucose tolerance (Kato, Kadota, Kitaura, Shimomura, unpublished observations). Moreover, the glucose tolerance deterioration in cirrhotic patients was improved by BCAA supplementation (7). These results clearly suggest that plasma BCAAs play an important role in glucose tolerance in mammals, and that BCAAs have therapeutic potential for the treatment of glucose intolerance.

\section{BCAAs and Brain Function}

Some amino acids function as neurotransmitters or their precursors in the brain. Glutamate is a very important excitatory neurotransmitter in the brain. BCAAs, especially leucine, play a vital role in the synthesis of glutamate in astrocytes, which are closely associated with brain capillaries, because leucine enters the brain from the blood more rapidly than other amino acids and provides $30-50 \%$ of all $\alpha$-amino groups of glutamate and glutamine (13).

It has been shown that a BDK defect results in chronic low levels of BCAAs in the plasma and tissues of mice. BCAA concentrations in the brains of BDK-KO mice were $\sim 30 \%$ of control mice, and neurological abnormalities were apparent from ongoing hind limb flexion and epileptic seizures after 6-7 mo of age (4), suggesting that BCAAs have an important role in neurological function. It is noteworthy that mice with a defect in autophagy in the central nervous system present a similar phenotype (i.e., hind limb flexion) (14). Very recently, patients with homozygous BDK mutations were identified; these patients showed markedly low levels of plasma BCAAs and suffered from autism with epilepsy $(15,16)$. Since some of the neurological phenotypes in BDK-KO mice were reversed by dietary supplementation with BCAAs, it may be possible to treat patients with BDK mutations with BCAA supplementation (15). 


\section{REFERENCES}

1) WHO/FAO/UNU. 2007. Amino acid requirements of adults. Protein and Amino Acid Requirements in Human Nutrition. Technical Reports Series 935, p 135159. WHO, Geneva.

2) Shimomura Y, Honda T, Shiraki M, Murakami T, Sato J, Kobayashi H, Mawatari K, Obayashi M, Harris RA. 2006. Branched-chain amino acid catabolism in exercise and liver disease. J Nutr 136: 250S-253S.

3) She P, Reid TM, Bronson SK, Vary TC, Hajnal A, Lynch CJ, Hutson SM. 2007. Disruption of BCATm in mice leads to increased energy expenditure associated with the activation of a futile protein turnover cycle. Cell Metab 6: 181-194.

4) Joshi MA, Jeoung NH, Obayashi M, Hattab EM, Brocken EG, Liechty EA, Kubek MJ, Vattem KM, Wek RC, Harris RA. 2006. Impaired growth and neurological abnormalities in branched-chain alpha-keto acid dehydrogenase kinase-deficient mice. Biochem J 400: 153-162.

5) Kimball SR, Jefferson LS. 2010. Control of translation initiation through integration of signals generated by hormones, nutrients, and exercise. J Biol Chem 285: 29027-29032.

6) Mizushima N, Komatsu M. 2011. Autophagy: renovation of cells and tissues. Cell 147: 728-741.

7) Kawaguchi T, Izumi N, Charlton MR, Sata M. 2011. Branched-chain amino acids as pharmacological nutrients in chronic liver disease. Hepatol 54: 1063-1070.

8) Sako K, Imamura Y, Nishimata H, Tahara K, Kubozono O, Tsubouchi H. 2003. Branched-chain amino acids supplements in the late evening decrease the frequency of muscle cramps with advanced hepatic cirrhosis. Hepatol Res 26: 327-329.

9) Nishitani S, Ijichi C, Takehana K, Fujitani S, Sonaka I. 2004. Pharmacological activities of branched-chain amino acids: specificity of tissue and signal transduction. Biochem Biophys Res Commun 313: 387-389.
10) Doi M, Yamaoka I, Nakayama M, Sugahara K, Yoshizawa F. 2007. Hypoglycemic effect of isoleucine involves increased muscle glucose uptake and whole body glucose oxidation and decreased hepatic gluconeogenesis. Am J Physiol Endocrinol Metab 292: E1683-E1693.

11) Nishitani S, Takehana K, Fujitani S, Sonaka I. 2005. Branched-chain amino acids improve glucose metabolism in rats with liver cirrhosis. Am J Physiol Gastrointest Liver Physiol 288: G1292-G300.

12) Kadota Y, Kazama S, Bajotto G, Kitaura Y, Shimomura Y. 2012. Clofibrate-induced reduction of plasma branchedchain amino acid concentrations impairs glucose tolerance in rats. JPEN J Parenter Enteral Nutr 36: 337-343.

13) Yudkoff M, Daikhin Y, Nissim I, Horyn O, Luhovyy B, Lazarow A, Nissim I. 2005. Brain amino acid requirements and toxicity: the example of leucine. J Nutr 135: 1531S-1538S.

14) Komatsu M, Waguri S, Chiba T, Murata S, Iwata J, Tanida I, Ueno T, Koike M, Uchiyama Y, Kominami E, Tanaka K. 2006. Loss of autophagy in the central nervous system causes neurodegeneration in mice. Nature 441: 880-884.

15) Novarino G, El-Fishawy P, Kayserili H, Meguid NA, Scott EM, Schroth J, Silhavy JL, Kara M, Khalil RO, BenOmran T, Ercan-Sencicek AG, Hashish AF, Sanders SJ, Gupta AR, Hashem HS, Matern D, Gabriel S, Sweetman L, Rahimi Y, Harris RA, State MW, Gleeson JG. 2012. Mutations in BCKD-kinase lead to a potentially treatable form of autism with epilepsy. Science 338: 394-397.

16) García-Cazorla A, Oyarzabal A, Fort J, Robles C, Castejón E, Ruiz-Sala P, Bodoy S, Merinero B, Lopez-Sala A, Dopazo J, Nunes V, Ugarte M, Artuch R, Palacín M, Rodríguez-Pombo P, Alcaide P, Navarrete R, Sanz P, FontLlitjós M, Vilaseca MA, Ormaizabal A, Pristoupilova A, Agulló SB. 2014. Two novel mutations in the BCKDK (branched-chain keto-acid dehydrogenase kinase) gene are responsible for a neurobehavioral deficit in two pediatric unrelated patients. Hum Mutat 35: 470-477. 